\title{
Efficient Linear Schemes with Unconditional Energy Stability for the Phase Field Model of Solid-State Dewetting Problems
}

\author{
Jie Chen ${ }^{1}$, Zhengkang $\mathrm{He}^{1}$, Shuyu Sun ${ }^{*}$, Shimin Guo ${ }^{1}$, and Zhangxin Chen ${ }^{3}$ \\ ${ }^{1}$ School of Mathematics and Statistics, Xi'an Jiaotong University, Shaanxi, P.R.China,710049 \\ ${ }^{2}$ Computational Transport Phenomena Laboratory, Division of Physical Science and \\ Engineering, King Abdullah University of Science and Technology, Thuwal 23955-6900, \\ Kingdom of Saudi Arabia \\ ${ }^{3}$ Schulich School of Engineering, University of Calgary, 2500 University Drive NW, Calgary, \\ AB, Canada T2N $1 \mathrm{~N} 4$
}

\begin{abstract}
In this paper, we study linearly first and second order in time, uniquely solvable and unconditionally energy stable numerical schemes to approximate the phase field model of solid-state dewetting problems based on the novel "scalar auxiliary variable" (SAV) approach, a new developed efficient and accurate method for a large class of gradient flows. The schemes are based on the first order Euler method and the second order backward differential formulas (BDF2) for time discretization, and finite element methods for space discretization. The proposed schemes are proved to be unconditionally stable and the discrete equations are uniquely solvable for all time steps. Various numerical experiments are presented to validate the stability and accuracy of the proposed schemes.

Key words: Phase field models; Solid-state dewetting; SAV; Energy stability; Surface diffusion; Finite element method
\end{abstract}

\section{Introduction}

Solid-state dewetting of thin films plays an important role in many engineering and industrial applications, such as microelectronics processing, formation of patterned silicides in electronic devices, production of catalysts for the growth of carbon and semiconductor nanowires $[1,3,4,5,6,7,18,19,28]$. In general, solid-state dewetting can be modeled as interfacial dynamic problems where the morphological evolution is controlled by the surface diffusion.

To understand the process of solid-state dewetting, many mathematical models have been developed, and numerical simulations based on these models have been carried out (cf. [1, 2, 28, 29] and the references therein). During the evolution, the film/vapor interface may experience complicated topological changes such as pinch-off, splitting and fattening, all of them make a great difficulty in the simulation of this interface evolution problem. A phase field model that can naturally captures the topological changes that occur during the morphological evolution

\footnotetext{
*Corresponding author(Email: shuyu.sun@kaust.edu.sa)
} 
and can be easily extended to high dimension spaces is presented in [1], where the spectral method with stabilized scheme is employed.

The idea of phase field approach dates back to the pioneering work of [22] and [30]. It has been successfully employed in many fields of science and engineering ever since. The phase field method uses an auxiliary variable $\phi$ (phase field function) to localize the phases and describe the interface by a layer of small thickness. The phase field function takes two different values (e.g., +1 and -1 ) in each of the two phases and varies smoothly across the interface. In the phase field model, the interface is considered as a transition layer over which a continuous, but steep change of some physical quantities occurs. The phase field model can be naturally derived from the variational principle, i.e., via minimizing the free energy of the total system. As a result, the derived system satisfies an energy dissipation law, which justifies its thermodynamic consistency and leads to a mathematically well-posed model. Moreover, the presence of the energy law serves as a guideline for the design of energy stable numerical schemes. The phase field method now becomes one of the major modeling and computational tools for the study of interfacial phenomena (cf. $[8,9,10,11,12,13,20,25,26])$, and the references therein).

From the numerical perspective, for phase field models, one main challenge in the numerical approximation is how to design unconditionally energy stable schemes which keep the energy dissipative in both semi-discrete and fully discrete forms. The preservation of the energy dissipation law is particularly important, and is critical to preclude the nonphysical numerical solutions. In fact, it has been observed that numerical schemes which do not respect the energy dissipation law may lead to large numerical errors, particular for long time simulation, so it is specially desirable to design numerical schemes that preserve the energy dissipation law at the discrete level. Another focus of developing numerical schemes to approximate the phase field models is to construct higher order time marching schemes. Under the requests of some degree of accuracy, higher order time marching schemes are usually preferable to lower order time marching schemes when we want to use larger time marching steps to achieve long time simulation. This fact motivates us to develop more accurate schemes. Moreover, it goes without saying that linear numerical schemes are more efficient than the nonlinear numerical schemes because the nonlinear schemes are expensive to solve.

In this paper, we study linearly first and second order accurate in time, uniquely solvable and unconditionally energy stable numerical schemes for solving the phase field model of solidstate dewetting problems based on the SAV approach which is applicable to a large class of gradient flows $[15,16]$. The schemes for gradient flows that introduce auxiliary variables are first presented in $[23,24]$ known as the invariant energy quadratization (IEQ) approach, where the auxiliary variable is a function. The essential idea of the SAV approach is to split the total free energy $\mathcal{E}(\phi)$ of gradient flows into two parts, written as

$$
\mathcal{E}(\phi)=\frac{1}{2}(\phi, \mathcal{L} \phi)+\mathcal{E}_{1}(\phi)
$$

where $\mathcal{L}$ is a symmetric non-negative linear operator contains the highest linear derivative terms 
in $\mathcal{E}$, and $\mathcal{E}_{1}(\phi) \geq C>0$ is the nonlinear term but with only lower order derivative than $\mathcal{L}$. Then the SAV approach transforms the nonlinear term $\mathcal{E}_{1}$ into quadratic form by introducing a scalar variable $r=\sqrt{\mathcal{E}_{1}}$ and the total free energy $\mathcal{E}$ can be rewritten as

$$
\mathcal{E}(\phi, r)=\frac{1}{2}(\phi, \mathcal{L} \phi)+r^{2}
$$

The rest of the paper is organized as follows. In section 2, we describe the phase field model of solid-state dewetting problems and the associated energy law. In Section 3, we develop linear numerical schemes with first order and second order accuracy in time for simulating the model respectively, and prove their unconditional energy stabilities and unconditionally unique solvability. In Section 4, various numerical experiments are performed to validate the accuracy and energy stability of the proposed schemes. Finally, some concluding remarks are given in Section 5 .

\section{The Governing System and Energy Law}

We now give a brief introduction to the phase-field model as is proposed in [1] that simulates the solid-state dewetting phenomenon of thin films and the morphological evolution of patterned islands on a solid substrate. If we consider that the free interface (surface between thin film phase and vapor phase) energy is isotropic, then the total free energy of the system is defined as follows

$$
\mathcal{E}(\phi)=\mathcal{E}_{F V}(\phi)+\mathcal{E}_{w}(\phi)=\int_{\Omega} f_{F V}(\phi) \mathrm{d} \mathbf{x}+\int_{\Gamma_{w}} f_{w}(\phi) \mathrm{d} s
$$

here $\Omega$ is a bounded domain in $\mathbb{R}^{2}$, with boundary $\partial \Omega$ that has an outward-pointing unit normal n. $\Gamma_{w} \subseteq \partial \Omega$ represents the solid surface (solid substrate) to where the thin film adhere, called as wall boundary. $\mathcal{E}_{F V}$ represents the free interface energy of the thin film and vapor phases, $\mathcal{E}_{w}$ represents the free energy on the solid surface and is called wall energy, and $f_{F V}$ and $f_{w}$ are the corresponding energy densities, respectively, defined as follows

$$
\begin{aligned}
f_{F V}(\phi) & =F(\phi)+\frac{\varepsilon^{2}}{2}|\nabla \phi|^{2} \\
f_{w}(\phi) & =\frac{\varepsilon\left(\phi^{3}-3 \phi\right)}{3 \sqrt{2}} \cos \theta_{s} .
\end{aligned}
$$

where $F(\phi)=\frac{1}{4}\left(\phi^{2}-1\right)^{2}$ is the well-known Ginzburg-Landau double-well potential, $\varepsilon$ is a positive constant related to the interface width, and $\theta_{s}$ is the prescribed contact angle between the free interface and the solid surface.

The governing equations of the system can be derived as a gradient flow of energy function (2.1) in $H^{-1}$ space:

$$
\begin{aligned}
\frac{\partial \phi}{\partial t} & =\nabla \cdot(M(\phi) \nabla \mu), & & \text { in } \Omega \\
\mu & =\frac{\delta \mathcal{E}}{\delta \phi}=\phi^{3}-\phi-\varepsilon^{2} \Delta \phi, & & \text { in } \Omega
\end{aligned}
$$


with the following boundary conditions

$$
\begin{aligned}
\varepsilon^{2} \frac{\partial \phi}{\partial \mathbf{n}}+f_{w}^{\prime} & =0, & \frac{\partial \mu}{\partial \mathbf{n}}=0, & & \text { on } \Gamma_{w} \\
\frac{\partial \phi}{\partial \mathbf{n}} & =0, & \frac{\partial \mu}{\partial \mathbf{n}}=0 . & & \text { on } \partial \Omega \backslash \Gamma_{w}
\end{aligned}
$$

The above system can be derived as a gradient flow of the total free energy functional $\mathcal{E}(\phi)$ with the dissipation mechanism $\phi_{t}=\nabla \cdot(M(\phi) \nabla \mu)$, where $M(\phi)=1-\phi^{2}$ is the mobility function chosen in [1] and $\mu$ is the chemical potential which is defined as the first variational derivative of the total free energy $\mathcal{E}$ with respect to the phase field variable $\phi$. The boundary condition $\frac{\partial \mu}{\partial \mathbf{n}}=0$ implies that the total mass is conservative:

$$
\frac{d}{d t} \int_{\Omega} \phi \mathrm{d} \mathbf{x}=\int_{\Omega} \phi_{t} \mathrm{~d} \mathbf{x}=\int_{\Omega} \nabla \cdot(M(\phi) \nabla \mu) \mathrm{d} \mathbf{x}=-\int_{\partial \Omega} M(\phi) \frac{\partial \mu}{\partial \mathbf{n}} \mathrm{d} s=0 .
$$

Moreover, the total free energy functional $\mathcal{E}(t)$ is dissipative:

$$
\begin{aligned}
\frac{d}{d t} \mathcal{E}(t) & =\int_{\Omega} F^{\prime}(\phi) \phi_{t}+\varepsilon^{2} \nabla \phi \cdot \nabla \phi_{t} \mathrm{~d} \mathbf{x}+\int_{\Gamma_{w}} f_{w}^{\prime}(\phi) \phi_{t} \mathrm{~d} s \\
& =\int_{\Omega} \mu \phi_{t} \mathrm{~d} \mathbf{x}+\int_{\Gamma_{w}}\left(\varepsilon^{2} \frac{\partial \phi}{\partial \mathbf{n}}+f_{w}^{\prime}(\phi)\right) \phi_{t} \mathrm{~d} s=\int_{\Omega} \mu M \triangle \mu \mathrm{d} \mathbf{x} \\
& =-\int_{\Omega} M \nabla \mu \cdot \nabla \mu \mathrm{d} \mathbf{x}+\int_{\partial \Omega} \mu M \frac{\partial \mu}{\partial \mathbf{n}} \mathrm{d} s \\
& =-\int_{\Omega} M|\nabla \mu|^{2} \mathrm{~d} \mathbf{x} \leq 0 .
\end{aligned}
$$

\section{Numerical schemes}

In this section, we construct several fully discrete numerical schemes to solve the dewetting problems in the framework of finite element methods, and prove their energy stabilities and unique solvability.

We aim to obtain some effective numerical schemes, in particular, the linear schemes. Inspired by the idea of the SAV approach, we split the total free energy $\mathcal{E}$ as follows,

$$
\begin{aligned}
\mathcal{E}(\phi)= & \frac{\varepsilon^{2}}{2}(\nabla \phi, \nabla \phi)+\frac{1}{4} \int_{\Omega}\left(\phi^{2}-1\right)^{2} \mathrm{~d} \mathbf{x}+\int_{\Gamma_{w}} \frac{\phi^{3}-3 \phi}{3 \sqrt{2}} \varepsilon \cos \theta_{s} \mathrm{~d} s \\
= & \frac{\varepsilon^{2}}{2}(-\Delta \phi, \phi)+\frac{\varepsilon^{2}}{2} \int_{\partial \Omega} \frac{\partial \phi}{\partial \mathbf{n}} \phi \mathrm{d} s+\frac{\beta}{2} \int_{\Omega} \phi^{2} \mathrm{~d} \mathbf{x} \\
& +\frac{1}{4} \int_{\Omega}\left(\phi^{2}-1-\beta\right)^{2} \mathrm{~d} \mathbf{x}+\int_{\Gamma_{w}} \frac{\phi^{3}-3 \phi}{3 \sqrt{2}} \varepsilon \cos \theta_{s} \mathrm{~d} s-\frac{1}{4} \int_{\Omega} \beta^{2}+2 \beta \mathrm{d} x \\
= & \frac{1}{2}(\phi, \mathcal{L} \phi)+\mathcal{E}_{1}(\phi)-\frac{1}{4} \int_{\Omega} \beta^{2}+2 \beta \mathrm{d} x,
\end{aligned}
$$

where $\beta$ is a positive constant to be chosen, and

$$
\begin{aligned}
(\phi, \mathcal{L} \phi) & =\varepsilon^{2}(-\Delta \phi, \phi)+\varepsilon^{2} \int_{\partial \Omega} \frac{\partial \phi}{\partial \mathbf{n}} \phi \mathrm{d} s+\beta \int_{\Omega} \phi^{2} \mathrm{~d} \mathbf{x}=\varepsilon^{2}(\nabla \phi, \nabla \phi)+\beta(\phi, \phi) \\
\mathcal{E}_{1}(\phi) & =\frac{1}{4} \int_{\Omega}\left(\phi^{2}-1-\beta\right)^{2} \mathrm{~d} \mathbf{x}+\int_{\Gamma_{w}} \frac{\phi^{3}-3 \phi}{3 \sqrt{2}} \varepsilon \cos \theta_{s} \mathrm{~d} s .
\end{aligned}
$$


Remark: The first term in $\mathcal{E}_{1}$ is nonnegative while the second term can be negative. Notice that the second term contains the interface thickness $\varepsilon$ as a factor, which is a tiny quantity. As a consequence, we require $\beta$ to be a positive constant so that the first term can control the second term. Thus the condition $\mathcal{E}_{1}(\phi) \geq C>0$ can be satisfied.

Since the governing equations of solid-state dewetting problems are obtained by minimizing the total free energy, we drop the constant $-\frac{1}{4} \int_{\Omega} \beta^{2}+2 \beta \mathrm{d} x$ in $\mathcal{E}(\phi)$. Then the total free energy becomes

$$
\mathcal{E}(\phi)=\frac{1}{2}(\phi, \mathcal{L} \phi)+\mathcal{E}_{1}(\phi),
$$

where $\mathcal{L}$ is a symmetric non-negative linear operator contains the highest linear derivative terms in $\mathcal{E}(\phi)$, and $\mathcal{E}_{1}(\phi) \geq C>0$ is the nonlinear term but with only lower order derivative than $\mathcal{L}$. To simplify notations, we still use $\mathcal{E}(\phi)$ to denote the modified total free energy. Then the gradient flow equations (2.4)-(2.5) can be written as

$$
\begin{aligned}
\frac{\partial \phi}{\partial t} & =\nabla \cdot\left(\left(1-\phi^{2}\right) \nabla \mu\right), \\
\mu & =\mathcal{L} \phi+U(\phi),
\end{aligned}
$$

where

$$
U(\phi)=\frac{\delta \mathcal{E}_{1}}{\delta \phi}
$$

is the first variational derivative of the nonlinear free energy $\mathcal{E}_{1}$ with respect to the phase field variable $\phi$.

As in $[15,16]$, a scalar auxiliary variable $r=\sqrt{\mathcal{E}_{1}}$ is introduced, then we rebuild the total free energy functional (3.4) as

$$
\mathcal{E}(\phi, r)=\frac{1}{2}(\phi, \mathcal{L} \phi)+r^{2},
$$

and accordingly we can rewrite the gradient flow equations (2.4)-(2.5) as follows

$$
\begin{aligned}
\frac{\partial \phi}{\partial t} & =\nabla \cdot\left(\left(1-\phi^{2}\right) \nabla \mu\right), \\
\mu & =\mathcal{L} \phi+\frac{r}{\sqrt{\mathcal{E}_{1}(\phi)}} U(\phi), \\
r_{t} & =\frac{1}{2 \sqrt{\mathcal{E}_{1}(\phi)}}\left\langle U(\phi), \phi_{t}\right\rangle .
\end{aligned}
$$

where,

$$
\left\langle U(\phi), \psi_{t}\right\rangle=\int_{\Omega}\left(\phi^{3}-\phi-\beta \phi\right) \psi_{t} \mathrm{~d} \mathbf{x}+\int_{\Gamma_{w}} \frac{\sqrt{2}}{2}\left(\phi^{2}-1\right) \varepsilon \cos \theta_{s} \psi_{t} \mathrm{~d} s .
$$

The boundary conditions are also (2.6)-(2.7), and the initial conditions are

$$
\phi(x, y, 0)=\phi_{0}, \quad r(0)=\sqrt{\mathcal{E}_{1}\left(\phi_{0}\right)} .
$$

Taking the inner products of the equations (3.9)-(3.11) with $\mu, \frac{\partial \phi}{\partial t}$ and $2 r$ respectively, the new system still follows an energy dissipative law:

$$
\frac{d}{d t} \mathcal{E}(\phi, r)=\frac{d}{d t}\left[(\phi, \mathcal{L} \phi)+r^{2}\right]=-(\mu, M(\phi) \mu) \leq 0 .
$$


Since finite element methods have the capability of handling complex geometries, we consider the fully discrete numerical schemes for solving the system (3.9)-(3.11) in the framework of finite element methods. Let $\mathcal{T}_{h}$ be a quasi-uniform triangulation of the domain $\Omega$ of mesh size $h$. We introduce the finite element space $S_{h}$ to approximate the Sobolev space $H^{1}(\Omega)$ based on the triangulation $\mathcal{T}_{h}$.

$$
S_{h}=\left\{v_{h} \in C(\Omega)\left|v_{h}\right|_{K} \in P_{r}, \forall K \in \mathcal{T}_{h}\right\},
$$

where $P_{r}$ is the space of polynomials of degree at most $r$ and $K$ is a triangular in $\mathcal{T}_{h}$. Denote the time step by $\delta t$ and set $t^{n}=n \delta t$. Here and after, for any function $g_{1}(x), g_{2}(x) \in L^{2}(\Omega)$, we denote the inner product as

$$
\left(g_{1}, g_{2}\right)=\int_{\Omega} g_{1}(x) g_{2}(x) \mathrm{d} x
$$

\subsection{The Linearly first order Scheme}

Firstly, we develop a first order time marching scheme for solving the system (3.9)-(3.11) by the semi-implicit backward Euler's method. Assuming that $\phi^{n}$ and $r^{n}$ are already known, we then solve $\phi^{n+1}$ and $r^{n+1}$ as follows:

$$
\begin{aligned}
& \frac{\phi^{n+1}-\phi^{n}}{\delta t}=\nabla \cdot\left(\left|1-\left(\phi^{n}\right)^{2}\right| \nabla \mu^{n+1}\right), \\
& \mu^{n+1}=\mathcal{L} \phi^{n+1}+\frac{r^{n+1}}{\sqrt{\mathcal{E}_{1}\left[\phi^{n}\right]}} U\left[\phi^{n}\right], \\
& r^{n+1}-r^{n}=\frac{1}{2 \sqrt{\mathcal{E}_{1}\left[\phi^{n}\right]}}\left\langle U\left[\phi^{n}\right], \phi^{n+1}-\phi^{n}\right\rangle .
\end{aligned}
$$

In the framework of the finite element methods, we now give the fully discrete scheme for the above discrete-in-time numerical system (3.16)-(3.18). Assuming that $\phi_{h}^{n}$ and $r_{h}^{n}$ are already known, we find $\left(\phi_{h}^{n+1}, \mu_{h}^{n+1}, r_{h}^{n+1}\right) \in S_{h} \times S_{h} \times \mathbb{R}^{+}$such that for all $\left(\nu_{h}, \psi_{h}\right) \in S_{h} \times S_{h}$ there hold

$$
\begin{aligned}
\left(\frac{\phi_{h}^{n+1}-\phi_{h}^{n}}{\delta t}, \nu_{h}\right) & =-\left(\left|1-\left(\phi_{h}^{n}\right)^{2}\right| \nabla \mu_{h}^{n+1}, \nabla \nu_{h}\right), \\
\left(\mu_{h}^{n+1}, \psi_{h}\right) & =\left(\mathcal{L} \phi_{h}^{n+1}, \psi_{h}\right)+\frac{r_{h}^{n+1}}{\sqrt{\mathcal{E}_{1}\left(\phi_{h}^{n}\right)}}\left\langle U\left(\phi_{h}^{n}\right), \psi_{h}\right\rangle, \\
r_{h}^{n+1}-r_{h}^{n} & =\frac{1}{2 \sqrt{\mathcal{E}_{1}\left(\phi_{h}^{n}\right)}}\left\langle U\left(\phi_{h}^{n}\right), \phi_{h}^{n+1}-\phi_{h}^{n}\right\rangle,
\end{aligned}
$$

where

$$
\begin{aligned}
\left(\mathcal{L} \phi_{h}^{n+1}, \psi_{h}\right) & =\varepsilon^{2}\left(-\Delta \phi_{h}^{n+1}, \psi\right)+\varepsilon^{2} \int_{\partial \Omega} \frac{\partial \phi_{h}^{n+1}}{\partial \mathbf{n}} \psi_{h} \mathrm{~d} s+\beta \int_{\Omega} \phi_{h}^{n+1} \psi_{h} \mathrm{~d} \mathbf{x} \\
& =\varepsilon^{2}\left(\nabla \phi_{h}^{n+1}, \nabla \psi_{h}\right)+\beta\left(\phi_{h}^{n+1}, \psi_{h}\right)
\end{aligned}
$$

and

$$
\left\langle U\left[\phi_{h}^{n+1}\right], \psi_{h}\right\rangle=\int_{\Omega}\left(\left(\phi_{h}^{n+1}\right)^{3}-\phi_{h}^{n+1}-\beta \phi_{h}^{n+1}\right) \psi_{h} \mathrm{~d} \mathbf{x}+\int_{\Gamma_{w}} \frac{\sqrt{2}}{2}\left(\left(\phi_{h}^{n+1}\right)^{2}-1\right) \varepsilon \cos \theta_{s} \psi_{h} \mathrm{~d} s .
$$


Remark: Taking $\nu_{h}=1$ in equation (3.19), we obtain the conservation of the total mass,

$$
\int_{\Omega} \phi_{h}^{n+1} \mathrm{~d} \mathbf{x}=\int_{\Omega} \phi_{h}^{n} \mathrm{~d} \mathbf{x}=\cdots=\int_{\Omega} \phi_{h}^{0} \mathrm{~d} \mathbf{x} .
$$

Theorem 3.1. Given $\left(\phi_{h}^{n}, r_{h}^{n}\right) \in S_{h} \times \mathbb{R}^{+}$, the system (3.19)-(3.21) admits a unique solution $\left(\phi_{h}^{n+1}, \mu_{h}^{n+1}, r_{h}^{n+1}\right) \in S_{h} \times S_{h} \times \mathbb{R}^{+}$at the time step $t^{n+1}$ for any $h>0$ and $\delta t>0$. Moreover, the solution satisfies a discrete energy law as follows

$$
\begin{aligned}
\mathcal{E}_{1 s t}^{n+1}-\mathcal{E}_{1 s t}^{n}+\frac{1}{2}\left(\phi_{h}^{n+1}-\phi_{h}^{n}, \mathcal{L}\left(\phi_{h}^{n+1}-\phi_{h}^{n}\right)\right) & +\left(r_{h}^{n+1}-r_{h}^{n}\right)^{2} \\
& =-\delta t\left(\left|1-\left(\phi_{h}^{n}\right)^{2}\right| \nabla \mu_{h}^{n+1}, \nabla \mu_{h}^{n+1}\right),
\end{aligned}
$$

where $\mathcal{E}_{1 \text { st }}^{n+1}$ is the modified energy

$$
\mathcal{E}_{1 s t}^{n}=\frac{1}{2}\left(\phi_{h}^{n}, \mathcal{L} \phi_{h}^{n}\right)+\left(r_{h}^{n}\right)^{2} .
$$

Thus the scheme is unconditionally stable.

Proof. By taking $\nu_{h}=\mu_{h}^{n+1}$ in equation (3.19), we obtain

$$
\left(\frac{\phi_{h}^{n+1}-\phi_{h}^{n}}{\delta t}, \mu_{h}^{n+1}\right)=-\left(\left|1-\left(\phi_{h}^{n}\right)^{2}\right| \nabla \mu_{h}^{n+1}, \nabla \mu_{h}^{n+1}\right),
$$

and by taking $\psi_{h}=\left(\phi_{h}^{n+1}-\phi_{h}^{n}\right) / \delta t$ in equations (3.20), we obtain

$$
\left(\mu_{h}^{n+1}, \frac{\phi_{h}^{n+1}-\phi_{h}^{n}}{\delta t}\right)=\left(\mathcal{L} \phi_{h}^{n+1}, \frac{\phi_{h}^{n+1}-\phi_{h}^{n}}{\delta t}\right)+\frac{r_{h}^{n+1}}{\sqrt{\mathcal{E}_{1}\left(\phi_{h}^{n}\right)}}\left\langle U\left(\phi_{h}^{n}\right), \frac{\phi_{h}^{n+1}-\phi_{h}^{n}}{\delta t}\right\rangle,
$$

By multiplying equation (3.21) with $2 r_{h}^{n+1} / \delta t$, we obtain

$$
2\left(r_{h}^{n+1}-r_{h}^{n}\right) r_{h}^{n+1}=\frac{r_{h}^{n+1}}{\sqrt{\mathcal{E}_{1}\left(\phi_{h}^{n}\right)}}\left\langle U\left(\phi_{h}^{n}\right), \phi_{h}^{n+1}-\phi_{h}^{n}\right\rangle .
$$

Adding equations (3.27)-(3.29) together, we obtain

$$
\left(\mathcal{L} \phi_{h}^{n+1}, \frac{\phi_{h}^{n+1}-\phi_{h}^{n}}{\delta t}\right)+2\left(r_{h}^{n+1}-r_{h}^{n}\right) r_{h}^{n+1}=-\left(\left|1-\left(\phi_{h}^{n}\right)^{2}\right| \nabla \mu_{h}^{n+1}, \nabla \mu_{h}^{n+1}\right),
$$

and in view of the following identities

$$
2(a-b, a)=|a|^{2}-|b|^{2}+|a-b|^{2}, \quad 2(a-b, b)=|a|^{2}-|b|^{2}-|a-b|^{2},
$$

we have the discrete energy law. Similarly, we can show the schemes (3.19)-(3.21) are uniquely solvable. Thus there exists a unique solution $\left(\phi_{h}^{n+1}, \mu_{h}^{n+1}, r_{h}^{n+1}\right)$ at time step $t^{n+1}$ for the linear system.

Remark: The convergence and error estimates of the SAV approach without the Lipschitz assumption is presented in [17], where the standard Ginzburg- Landau free energy together with periodic boundary conditions is considered. Then the authors extend their result to several other gradient flows. However, this framework doesn't work for the dewetting problem whose free energy contains a boundary integral (the wall energy). If there is boundary integral in the free energy, the $H_{1}$ bound, $H_{2}$ bound and error estimate for the numerical solutions are unavailable. 


\subsection{The Linearly second order Scheme}

We now develop linearly semi-implicit second order numerical scheme based on the backward differentiation formula (BDF2). Assuming that $\phi^{n}, \phi^{n-1}, r^{n}$ and $r^{n-1}$ are already known, we compute $\phi^{n+1}$ and $r^{n+1}$ as follows:

$$
\begin{aligned}
& \frac{3 \phi^{n+1}-4 \phi^{n}+\phi^{n-1}}{2 \delta t}=\nabla \cdot\left(\left|1-\left(\bar{\phi}^{n+1}\right)^{2}\right| \nabla \mu^{n+1}\right), \\
& \mu^{n+1}=\mathcal{L} \phi^{n+1}+\frac{r^{n+1}}{\sqrt{\mathcal{E}_{1}\left[\bar{\phi}^{n+1}\right]}} U\left[\bar{\phi}^{n+1}\right], \\
& 3 r^{n+1}-4 r^{n}+r^{n-1}=\frac{1}{2 \sqrt{\mathcal{E}_{1}\left[\bar{\phi}^{n+1}\right]}}\left\langle U\left[\bar{\phi}^{n+1}\right], 3 \phi^{n+1}-4 \phi^{n}+\phi^{n-1}\right\rangle,
\end{aligned}
$$

where, $\bar{\phi}^{n+1}=2 \phi^{n}-\phi^{n-1}$ is the explicit approximation of $\phi\left(t^{n+1}\right)$ with local truncation error of $O\left(\delta t^{2}\right)$.

In the framework of the finite element methods, we now give the fully discrete second order numerical scheme for the discrete-in-time numerical scheme (3.32)-(3.34). Assuming that $\phi_{h}^{n-1}$, $r_{h}^{n-1}, \phi_{h}^{n}$ and $r_{h}^{n}$ are already known, we find $\left(\phi_{h}^{n+1}, \mu_{h}^{n+1}, r_{h}^{n+1}\right) \in S_{h} \times S_{h} \times \mathbb{R}^{+}$such that for all $\left(\nu_{h}, \psi_{h}\right) \in S_{h} \times S_{h}$ there hold

$$
\begin{aligned}
& \left(\frac{3 \phi_{h}^{n+1}-4 \phi_{h}^{n}+\phi_{h}^{n-1}}{2 \delta t}, \nu_{h}\right)=-\left(\left|1-\left(\bar{\phi}_{h}^{n+1}\right)^{2}\right| \nabla \mu_{h}^{n+1}, \nabla \nu_{h}\right) \\
& \left(\mu_{h}^{n+1}, \psi_{h}\right)=\left(\mathcal{L} \phi_{h}^{n+1}, \psi_{h}\right)+\frac{r_{h}^{n+1}}{\sqrt{\mathcal{E}_{1}\left(\bar{\phi}_{h}^{n+1}\right)}}\left\langle U\left(\bar{\phi}_{h}^{n+1}\right), \psi_{h}\right\rangle, \\
& 3 r_{h}^{n+1}-4 r_{h}^{n}+r_{h}^{n-1}=\frac{1}{2 \sqrt{\mathcal{E}_{1}\left(\bar{\phi}_{h}^{n+1}\right)}}\left\langle U\left(\bar{\phi}_{h}^{n+1}\right), 3 \phi_{h}^{n+1}-4 \phi_{h}^{n}+\phi_{h}^{n-1}\right\rangle,
\end{aligned}
$$

where $\bar{\phi}_{h}^{n+1}=2 \phi_{h}^{n}-\phi_{h}^{n-1}$,

$$
\begin{aligned}
\left(\mathcal{L} \phi_{h}^{n+1}, \psi_{h}\right) & =\varepsilon^{2}\left(-\Delta \phi_{h}^{n+1}, \psi\right)+\varepsilon^{2} \int_{\partial \Omega} \frac{\partial \phi_{h}^{n+1}}{\partial \mathbf{n}} \psi_{h} \mathrm{~d} s+\beta \int_{\Omega} \phi_{h}^{n+1} \psi_{h} \mathrm{~d} \mathbf{x} \\
& =\varepsilon^{2}\left(\nabla \phi_{h}^{n+1}, \nabla \psi_{h}\right)+\beta\left(\phi_{h}^{n+1}, \psi_{h}\right),
\end{aligned}
$$

and

$$
\left\langle U\left[\bar{\phi}_{h}^{n+1}\right], \psi_{h}\right\rangle=\int_{\Omega}\left(\left(\bar{\phi}_{h}^{n+1}\right)^{3}-\bar{\phi}_{h}^{n+1}-\beta \bar{\phi}_{h}^{n+1}\right) \psi_{h} \mathrm{~d} \mathbf{x}+\int_{\Gamma_{w}} \frac{\sqrt{2}}{2}\left(\left(\bar{\phi}_{h}^{n+1}\right)^{2}-1\right) \varepsilon \cos \theta_{s} \psi_{h} \mathrm{~d} s .
$$

Remark: The second order scheme (3.35)-(3.37) is a two step method. We can solve for $\phi_{h}^{1}$ and $r_{h}^{1}$ through the first order scheme (3.19)-(3.21). Similarly by taking $\nu_{h}=1$ in equation (3.35), we obtain the conservation of the total mass,

$$
\int_{\Omega} \phi_{h}^{n+1} \mathrm{~d} \mathbf{x}=\int_{\Omega} \phi_{h}^{n} \mathrm{~d} \mathbf{x}=\cdots=\int_{\Omega} \phi_{h}^{0} \mathrm{~d} \mathbf{x}
$$

Theorem 3.2. Given $\left(\phi_{h}^{n}, r_{h}^{n}\right) \in S_{h} \times \mathbb{R}^{+}$, the system (29)-(31) admits a unique solution $\left(\phi_{h}^{n+1}, \mu_{h}^{n+1}, r_{h}^{n+1}\right) \in S_{h} \times S_{h} \times \mathbb{R}^{+}$at the time step $t^{n+1}$ for any $h>0$ and $\delta t>0$. Moreover, 
the solution satisfies a discrete energy law as follows

$$
\begin{aligned}
\mathcal{E}_{2 n d}^{n+1, n}-\mathcal{E}_{2 n d}^{n, n-1} & +\frac{1}{4}\left(\phi_{h}^{n+1}-2 \phi_{h}^{n}+\phi_{h}^{n-1}, \mathcal{L}\left(\phi_{h}^{n+1}-2 \phi_{h}^{n}+\phi_{h}^{n-1}\right)\right) \\
& +\frac{1}{2}\left(r_{h}^{n+1}-2 r_{h}^{n}+r_{h}^{n-1}\right)^{2}=-\delta t\left(\left|1-\left(\bar{\phi}_{h}^{n+1}\right)^{2}\right| \nabla \mu_{h}^{n+1}, \nabla \mu_{h}^{n+1}\right)
\end{aligned}
$$

where $\mathcal{E}_{2 n d}^{n+1, n}$ is the modified energy

$$
\mathcal{E}_{2 n d}^{n+1, n}=\frac{1}{4}\left(\left(\phi_{h}^{n+1}, \mathcal{L} \phi_{h}^{n+1}\right)+\left(2 \phi_{h}^{n+1}-\phi_{h}^{n}, \mathcal{L}\left(2 \phi_{h}^{n+1}-\phi_{h}^{n}\right)\right)+\frac{1}{2}\left(\left(r_{h}^{n+1}\right)^{2}+\left(2 r_{h}^{n+1}-r_{h}^{n}\right)^{2}\right)\right) .
$$

Proof. By taking $\nu_{h}=\mu_{h}^{n+1}$ in equation (3.35), we obtain

$$
\left(\frac{3 \phi_{h}^{n+1}-4 \phi_{h}^{n}+\phi_{h}^{n-1}}{2 \delta t}, \mu_{h}^{n+1}\right)=-\left(\left|1-\left(\bar{\phi}_{h}^{n+1}\right)^{2}\right| \nabla \mu_{h}^{n+1}, \nabla \mu_{h}^{n+1}\right) .
$$

By taking $\psi_{h}=\left(3 \phi_{h}^{n+1}-4 \phi_{h}^{n}+\phi_{h}^{n-1}\right) / \delta t$ in equations (3.36), we obtain

$$
\begin{aligned}
\left(\mu_{h}^{n+1}, \frac{3 \phi_{h}^{n+1}-4 \phi_{h}^{n}+\phi_{h}^{n-1}}{2 \delta t}\right)= & \left(\mathcal{L} \phi_{h}^{n+1}, \frac{3 \phi_{h}^{n+1}-4 \phi_{h}^{n}+\phi_{h}^{n-1}}{2 \delta t}\right) \\
& +\frac{r_{h}^{n+1}}{\sqrt{\mathcal{E}_{1}\left(\bar{\phi}_{h}^{n+1}\right)}}\left\langle U\left(\bar{\phi}_{h}^{n+1}\right), \frac{3 \phi_{h}^{n+1}-4 \phi_{h}^{n}+\phi_{h}^{n-1}}{2 \delta t}\right\rangle .
\end{aligned}
$$

By multiplying equation (3.37) with $2 r_{h}^{n+1} / \delta t$, we obtain

$$
2\left(3 r_{h}^{n+1}-4 r_{h}^{n}+r_{h}^{n-1}\right) r_{h}^{n+1}=\frac{r_{h}^{n+1}}{\sqrt{\mathcal{E}_{1}\left(\bar{\phi}_{h}^{n+1}\right)}}\left\langle U\left(\bar{\phi}_{h}^{n+1}\right), 3 \phi_{h}^{n+1}-4 \phi_{h}^{n}+\phi_{h}^{n-1}\right\rangle .
$$

Integrating the equations (3.42)-(3.44) together, we obtain

$\left(\mathcal{L} \phi_{h}^{n+1}, \frac{3 \phi_{h}^{n+1}-4 \phi_{h}^{n}+\phi_{h}^{n-1}}{2 \delta t}\right)+2\left(3 r_{h}^{n+1}-4 r_{h}^{n}+r_{h}^{n-1}\right) r_{h}^{n+1}=-\left(\left|1-\left(\bar{\phi}_{h}^{n+1}\right)^{2}\right| \nabla \mu_{h}^{n+1}, \nabla \mu_{h}^{n+1}\right)$.

Applying the following identity

$$
\begin{aligned}
2\left(3 a^{k+1}-4 a^{k}+a^{k-1}, a^{k+1}\right) & =\left|a^{k+1}\right|^{2}+\left|2 a^{k+1}-a^{k}\right|^{2}+\left|a^{k+1}-2 a^{k}+a^{k-1}\right|^{2} \\
& -\left|a^{k}\right|^{2}-\left|2 a^{k}-a^{k-1}\right|^{2},
\end{aligned}
$$

we can obtain the discrete energy law. Similarly, we can show the schemes (3.35)-(3.37) are uniquely solvable. Thus there exists a unique solution $\left(\phi_{h}^{n+1}, \mu_{h}^{n+1}, r_{h}^{n+1}\right)$ at time step $t^{n+1}$ for this linear system.

\section{Numerical experiments}

In this section, we present some numerical experiments to validate the accuracy, reliability and stability of the fully discrete numerical schemes presented in this paper. For simplicity, we use the conforming $P_{1}$ finite element in space $S_{h}$ to approximate phase field variable $\phi_{h}$ and chemical potential $\mu_{h}$, respectively. The algorithms are implemented in MATLAB using the software library $i$ FEM [27]. 


\section{1. convergence test}

In this subsection, we provide some numerical evidence to show the first order temporal accuracy for the numerical scheme (3.19)-(3.21) and the second order temporal accuracy for the numerical scheme (3.35)-(3.37) by Cauchy convergence test [32]. For all our experiments in this subsection, the computational domain is taken as a rectangle $\Omega=[-1,1] \times[0,1]$, and the wall boundary $\Gamma_{w}$ (surface) is the bottom of the rectangle domain, defined as

$$
\Gamma_{w}=\{(x, y) \mid-1<x<1, y=0\} .
$$

The domain $\Omega$ is triangulated by a structured mesh with uniform $2^{k+1}$ grid points in the $x$ direction and uniform $2^{k}$ grid points in the $y$ direction, for $\mathrm{k}$ from 4 to 8 . The initial data of the phase field variable is taken to be

$$
\phi(x, y, 0)=\tanh \left(\frac{0.25-\sqrt{x^{2}+y^{2}}}{\sqrt{2} \varepsilon}\right), \quad \phi_{h}^{0}=\mathcal{I}_{h}\{\phi(x, y, 0)\}
$$

where, $\mathcal{I}_{h}: H^{2}(\Omega) \rightarrow S_{h}$ is the standard nodal interpolation operator, and other parameters are taken to be $\varepsilon=0.1, \beta=5$. We use five different contact angles $\theta_{s}=\pi, \theta_{s}=3 \pi / 4$, $\theta_{s}=\pi / 2, \theta_{s}=\pi / 4, \theta_{s}=0$ to test the convergence rate, respectively. To get around the fact that we do not have possession of explicit exact solution, we compute the rate at which the Cauchy difference $\phi_{h}^{k}-\phi_{h}^{k-1}$ converges to zero. To check the second order convergence rate of the scheme (3.35)-(3.37), the final time is taken to be $T=0.1$ and the time step is taken to be $\delta t=0.2 h$. Since the $P_{1}$ finite element approximation is used for the phase field variable $\phi_{h}$, the $L^{2}$ norm of the Cauchy difference error $\left\|\phi_{h}^{k}-\phi_{h}^{k-1}\right\|_{2}$ is expected to converge to zero at the rate of second order err $=O\left(\delta t^{2}\right)+O\left(h^{2}\right)=O\left(h^{2}\right)$. The Cauchy errors and the relative convergence rates are presented in Table 4.1 which shows the second order convergence for five different contact angles, respectively.

Table 4.1:: Cauchy convergence test for the second order linear numerical scheme (3.35)-(3.37) with the initial condition (44), parameters are $\varepsilon=0.1$ and $\beta=5$, errors are measured in $L^{2}$ norm; $2^{k+1}$ and $2^{k}$ grid points in the $x$ and $y$ direction for $\mathrm{k}$ from 4 to 8 , five different contact angles $\theta_{s}=\pi, \theta_{s}=3 \pi / 4, \theta_{s}=\pi / 2, \theta_{s}=\pi / 4, \theta_{s}=0$ are tested respectively

\begin{tabular}{cccccccc}
\hline$\theta$ & $16-32$ & Cvg.rate & $32-64$ & Cvg.rate & $64-128$ & Cvg.rate & $128-256$ \\
\hline$\pi$ & 0.0063 & 1.9480 & 0.0016 & 2.0777 & $3.8837 \mathrm{e}-004$ & 2.1738 & $8.9332 \mathrm{e}-005$ \\
$3 \pi / 4$ & 0.0063 & 1.9505 & 0.0016 & 2.0246 & $3.9578 \mathrm{e}-004$ & 2.0869 & $9.4824 \mathrm{e}-005$ \\
$\pi / 2$ & 0.0061 & 1.9379 & 0.0016 & 1.9797 & $3.9895 \mathrm{e}-004$ & 1.9978 & $9.9849 \mathrm{e}-005$ \\
$\pi / 4$ & 0.0066 & 1.9218 & 0.0017 & 1.9882 & $4.2918 \mathrm{e}-004$ & 2.0253 & $1.0596 \mathrm{e}-004$ \\
0 & 0.0069 & 1.9084 & 0.0018 & 1.9850 & $4.5246 \mathrm{e}-004$ & 2.0179 & $1.1211 \mathrm{e}-004$ \\
\hline
\end{tabular}

\subsection{Solid-state dewetting simulation over flat surface}

In this subsection, we present some two-dimensional simulations for the solid-state dewetting problems using the schemes (3.35)-(3.37). Numerical simulations in [7] suggest that in 
order to accurately capture the interfacial dynamics, at least four elements are needed across the interfacial region of thickness $\sqrt{2} \varepsilon$. We explore adaptive mesh refinement algorithm of the software library $i$ FEM [27] with the finest element size $h=1 / 256$ to improve the computational efficiency. At each time step, local error indicators are calculated heuristically. These error indicators then govern the refinement and coarsening process of the grid. The heuristic strategy is to refine the transition zones while coarsening the phases. To identify the transition zone, several approaches are proved to be equally suitable. One could either refine regions where the norm of the gradient of the solution is bigger than a certain threshold, or where the concentration is far from the minima of the nonlinearity $F(\phi)=\frac{1}{4}\left(\phi^{2}-1\right)^{2}$.

For all our experiments in this subsection, the computational domain is taken as a rectangle $\Omega=[-1,1] \times[0,1]$, and the wall boundary $\Gamma_{w}$ (surface) is the bottom of the rectangle domain, defined as

$$
\Gamma_{w}=\{(x, y) \mid-1<x<1, y=0\} .
$$

The numerical simulations were terminated when the thin film shape achieves the equilibrium state, defined numerically when the discrete $L_{2}$ norm $\left\|\phi_{h}^{n+1}-\phi_{h}^{n}\right\|_{2} \leq 10^{-5}$ is first achieved.

Example 1: The initial state of the thin film is taken to be a small rectangle:

$$
\phi(x, y, 0)= \begin{cases}1 & \text { if }-0.5 \leq x \leq 0.5 \text { and } 0 \leq y \leq 0.2 \\ -1 & \text { otherwise }\end{cases}
$$

the computational parameters are taken as: $\varepsilon=0.01, \beta=5$ and $\delta t=1 / 128$.

We examine the evolution of thin film under eight different prescribed contact angles: $\theta_{s}=\pi, \theta_{s}=5 \pi / 6, \theta_{s}=3 \pi / 4, \theta_{s}=\pi / 2, \theta_{s}=\pi / 3, \theta_{s}=\pi / 4, \theta_{s}=\pi / 6$ and $\theta_{s}=0$, respectively. The evolutions of film profiles are shown in Fig. 4.1. The film profiles evolve towards the equilibrium state quickly and the prescribed contact angles are achieved rapidly. Then these angles are maintained throughout the island morphology evolutions. For example, for the extreme case $\theta_{s}=\pi$, the film profile converges to a full circle quickly and maintain this shape throughout the simulation. For the complete wetting case $\theta_{s}=0$, the equilibrium is achieved rapidly and this continuous film is maintained throughout the film profile evolution.

We plot dissipative curves of the modified free energy in Fig. 4.2 using ten different time steps $\delta t=3.2 e-5,1.6 e-5,8 e-6,4 e-6,2 e-6,1 e-6,5 e-7,2.5 e-7,1.25 e-7,6.25 e-8$ with the prescribed contact angle $\theta_{s}=\pi / 2$. We observe that the energy decreases at all times, which confirms that our algorithm is unconditionally stable, as predicted by the theory. The total free energy decreases dramatically at the early stage of the simulation and keeps unchanged in the remaining time. This coincides the results of film profile evolution. Moreover, the energy curves converge to one curve as the time step decreases. This fact illustrates the convergence of the proposed schemes numerically.

Since the auxiliary variable is introduced in the SAV approach, the modified energy is equivalent to the original energy in PDE level but different in discrete level. We plot the original energy and the modified energy at three different time steps $\delta t=1.6 e-5,1 e-6,6.25 e-8$ with 


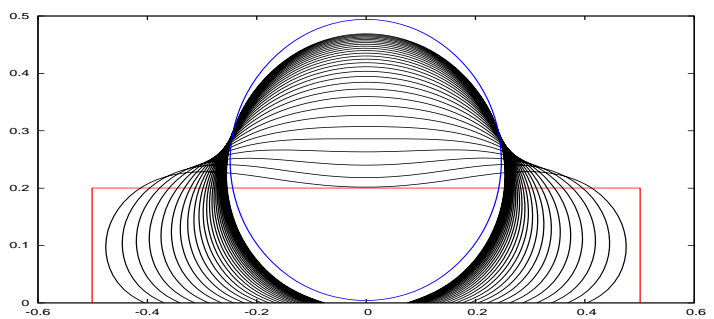

(a)

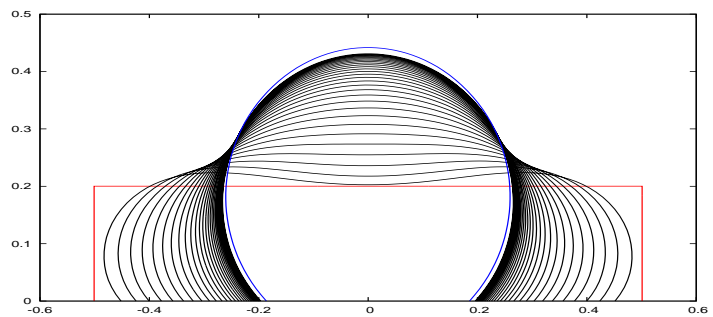

(c)

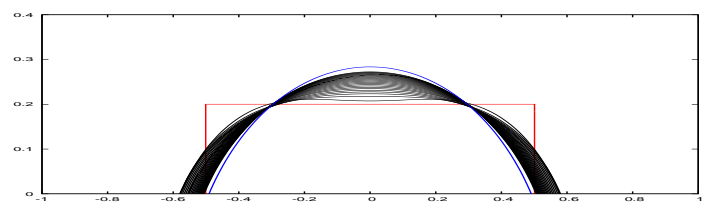

(e)

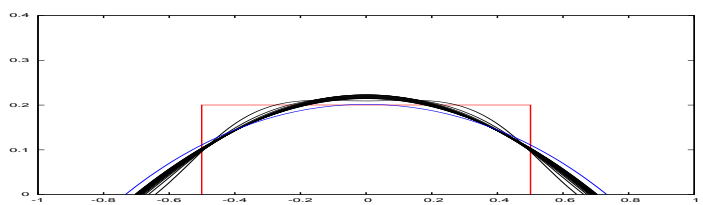

$(\mathrm{g})$

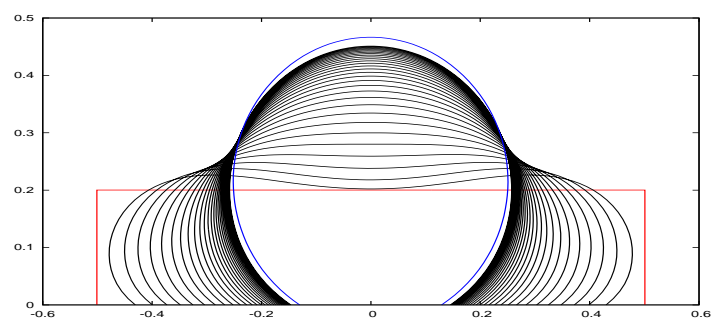

(b)

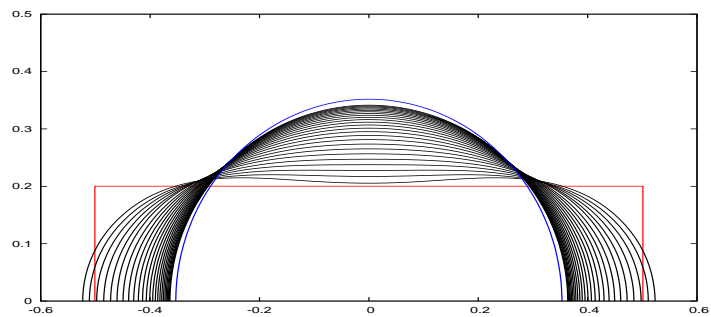

(d)

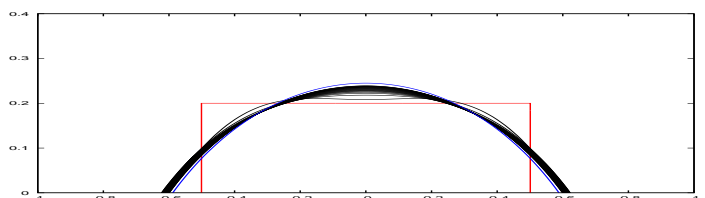

(f)

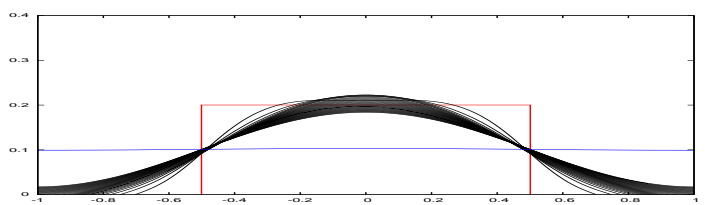

(h)

Fig. 4.1.: The evolution of the thin film for eight different prescribed contact angles: (a) $\theta_{s}=\pi$, (b) $\theta_{s}=5 \pi / 6$, (c) $\theta_{s}=3 \pi / 4,(\mathrm{~d}) \theta_{s}=\pi / 2,(\mathrm{e}) \theta_{s}=\pi / 3,(\mathrm{f}) \theta_{s}=\pi / 4,(\mathrm{~g}) \theta_{s}=\pi / 6$, (h) $\theta_{s}=0$. The film profiles are shown every 2500 time steps (labeled as black lines). The red line and blue line represent the initial and numerical equilibrium states, respectively.

the prescribed contact angle $\theta_{s}=\pi / 2$ in Fig. 4.3. We can see that the modified free energy converges to the original free energy as the time step decreases.

Example 2: The initial film occupies $x \leq-0.05$ and $x \geq 0.05$, and has a thickness of 0.1 .

$$
\phi(x, y, 0)= \begin{cases}+1, & x \in[-1,-0.05] \cup[0.05,1] \text { and } y \in[0,0.1] \\ -1, & \text { otherwise. }\end{cases}
$$

the computational parameters are taken as: $\varepsilon=0.005, \beta=5$ and $\delta t=1 / 128$.

We examine the evolution of thin film containing a small hole at its center under two different prescribed contact angles: $\theta_{s}=5 \pi / 6$ and $\theta_{s}=\pi / 6$, respectively. The results are shown in Fig. 4.4, for the $\theta_{s}=5 \pi / 6$ case, the hole grows and eventually achieves the equilibrium circular shape. However, for $\theta_{s}=\pi / 6$ the initial hole closes, the valley where the hole is located disappears and the film approaches a metastable flat morphology. The numerical results are 


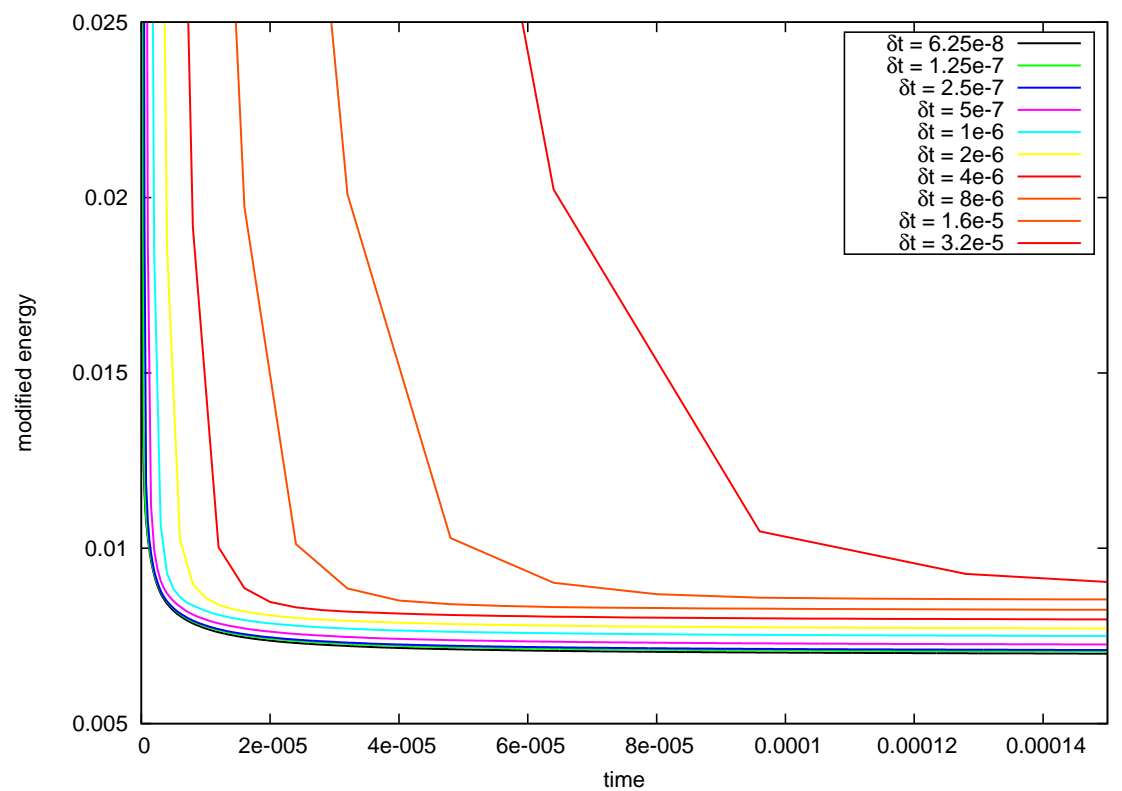

Fig. 4.2.: Time evolution of the free energy functional for ten different time steps of $\delta t=3.2 e-5,1.6 e-5,8 e-6,4 e-$ $6,2 e-6,1 e-6,5 e-7,2.5 e-7,1.25 e-7,6.25 e-8$ with the prescribed contact angle $\theta_{s}=\pi / 2$. The energy curves show the decays for all time steps, which confirms that our algorithm is unconditionally stable.

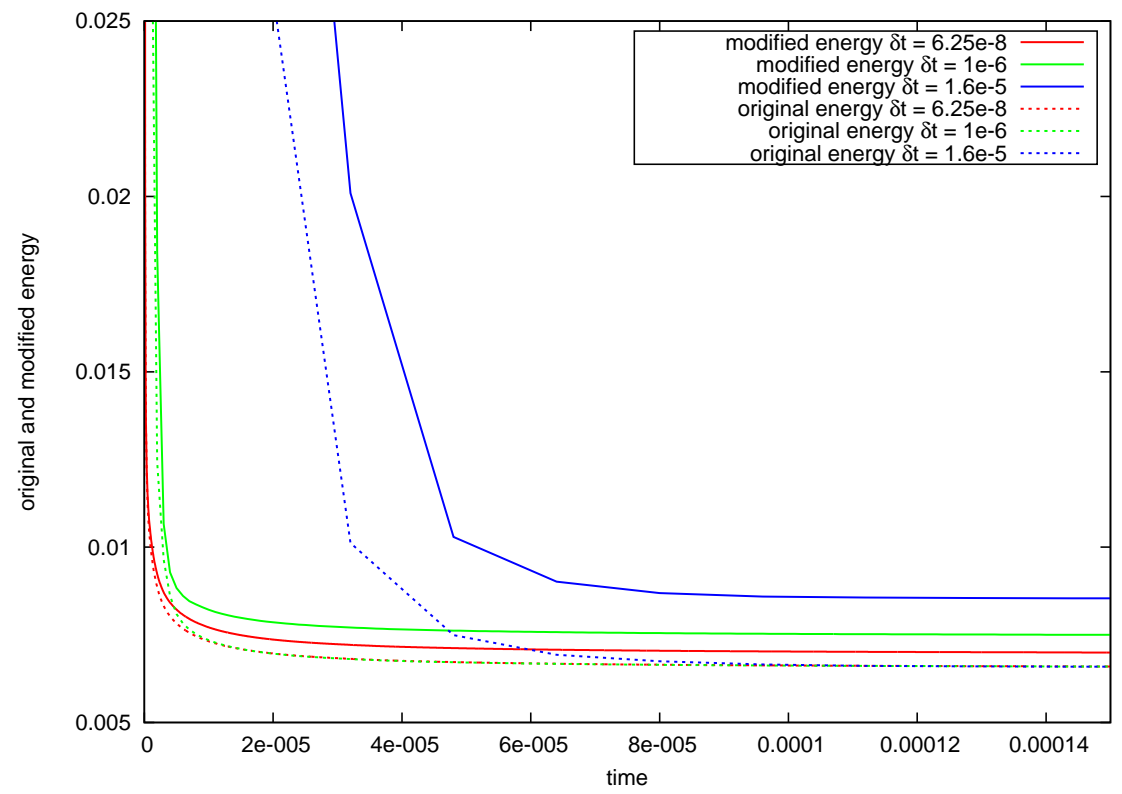

Fig. 4.3.: Time evolution of the original and modified free energy functional for three different time steps of $\delta t=$ $1.6 e-5,1 e-6,6.25 e-8$ with the prescribed contact angle $\theta_{s}=\pi / 2$.

consistent with the results in [1]. 

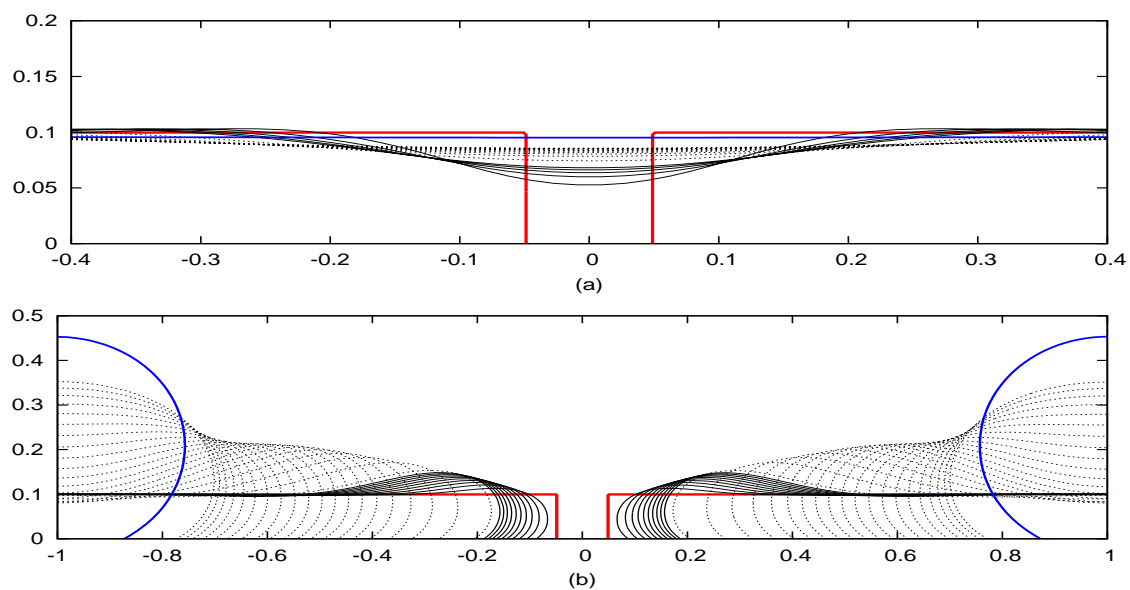

Fig. 4.4.: The evolution of a thin film containing a hole in its center for two different prescribed contact angles: (a) $\theta_{s}=\pi / 6$, (b) $\theta_{s}=5 \pi / 6$. The film profiles are shown every 200 time steps (labeled as solid black lines) and every 2500 time steps (labeled as dotted black lines). The red line and blue line represent the initial and numerical equilibrium states, respectively.

\subsection{Solid-state dewetting simulation over curved surface}

In this subsection, the solid-state dewetting of a thin film on a curved substrate is simulated using the second order schemes (3.35)-(3.37). As shown in Fig. 4.5, the computational domain is $[x, y] \in[-1,1] \times[0,1]$, a thin film is initially placed on a cylinder substrate surface. The film occupies $-0.5 \leq x \leq 0.5$, and has a thickness of 0.1363 . The radius of the bottom surface is 3.8637 and position of the circle center is $(0,-3.7321)$. The other parameters employed in this simulation are $\varepsilon=0.01, \delta t=0.01, \beta=5$. The numerical simulations are terminated when the thin film shape achieves the equilibrium state, defined numerically when the discrete $L_{2}$ norm $\left\|\phi_{h}^{n+1}-\phi_{h}^{n}\right\|_{2} \leq 10^{-5}$ is first achieved.

We set the prescribed contact angle $\theta_{s}$ to be $2 \pi / 3$ and $\pi / 3$ respectively in the simulation. As shown in Fig. 4.6 and 4.7, with the different prescribed contact angles, the thin film evolves towards different directions from the initial position. We also plot dissipative curves of the modified free energy in Fig. 4.8 for two different prescribed contact angles $\theta_{s}=2 \pi / 3$ and $\theta_{s}=\pi / 3$ with time step $\delta t=0.01$. The energy curves show the decays for all prescribed contact angle, which confirms that our algorithm is unconditionally stable, as predicted by the theory.

\section{Conclusions}

In this paper, we present linearly first and second order in time, uniquely solvable and unconditionally energy stable schemes for solving the solid-state dewetting problems based on the novel SAV approach. We verify numerically that our schemes are up to second order accurate in time. The adaptive strategy is used to improve the efficiency of the algorithm. Numerical examples are presented to illustrate the stability and accuracy of the proposed schemes. 


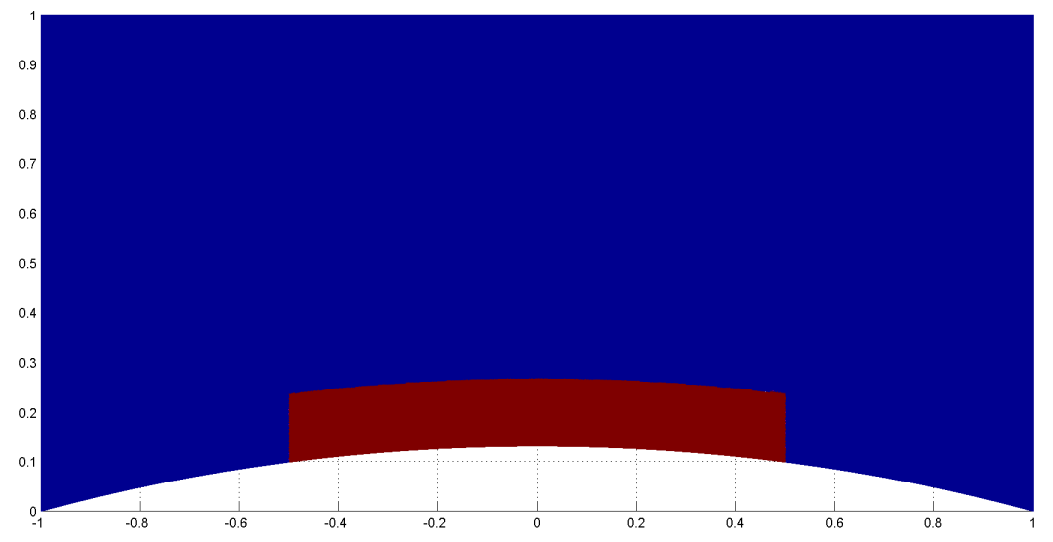

Fig. 4.5.: The initial configuration of the solid-state dewetting problems: a thin film on the curved surface.

\section{Acknowledgement}

The work is supported by the National Natural Science Foundation of China (No.11401467), China Postdoctoral Science Foundation (No. 2013M542334. and No. 2015T81012), and Natural Science Foundation of Shaanxi Province (No. 2015JQ1012). The work is also supported in part by funding from King Abdullah University of Science and Technology (KAUST) through the grant BAS/1/1351-01-01.

\section{References}

[1] Jiang, W., Bao, W.: Phase field approach for simulating solid-state dewetting problems. Acta Materialia. 60, 5578-5592 (2012).

[2] Wang, Y., Jiang, W.: Sharp interface model for solid-state dewetting problems with weakly anisotropic surface energies[J]. Physical Review B. 2015, 91(4): 045303.

[3] Yi Wang, Shuyu Sun, Liang Gong, Bo Yu. A globally mass-conservative method for dualcontinuum gas reservoir simulation. Journal of Natural Gas Science and Engineering, 2018, 53C: $301-316$

[4] Yi Wang, Bo Yu, Ye Wang. Acceleration of gas reservoir simulation using proper orthogonal decomposition. Geofluids, 2018, Article ID 8482352: 1-15.

[5] Yi Wang, Shuyu Sun, Bo Yu. Acceleration of gas flow simulations in dual-continuum porous media based on the mass-conservation POD method. Energies, 2017, 10(1380): 1-17.

[6] Yi Wang, Shuyu Sun. Direct calculation of permeability by high-accurate finite difference and numerical integration methods. Communications in Computational Physics, 2016, 20(2): 405-440.

[7] Mordehai, D., Kazakevich, M.: Nanoindentation size effect in single-crystal nanoparticles and thin films: A comparative experimental and simulation study. Acta Materialia. 59, 2309-2321 (2011).

[8] X. P. Wang, T. Qian, and P. Sheng, Moving contact line on chemically patterned surfaces, J. Fluid Mech. 605(2008) pp. 59-78.

[9] M. Gao, and X. P. Wang, A gradient stable scheme for a phase field model for the moving contact line problem, J. Comput. Phys. 231 (2012) pp. 1372-1386. 


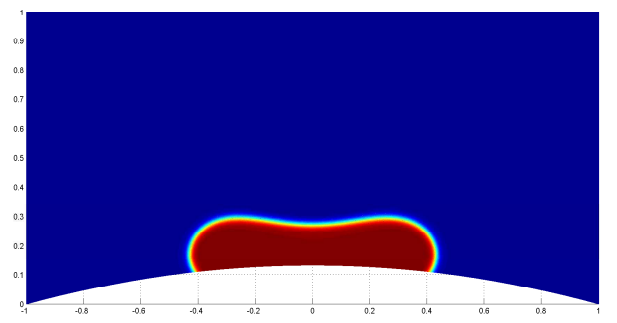

(a) $\mathrm{t}=25$

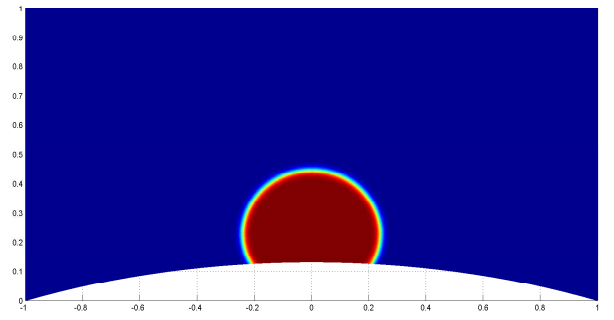

(c) $\mathrm{t}=200$

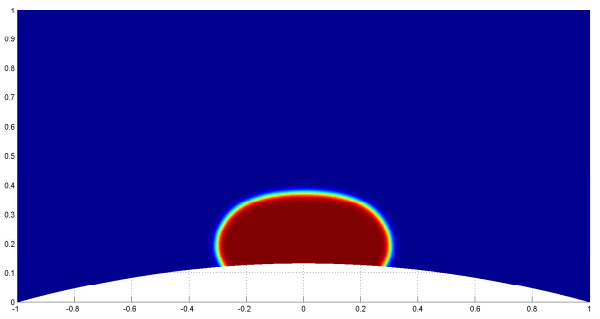

(b) $\mathrm{t}=100$

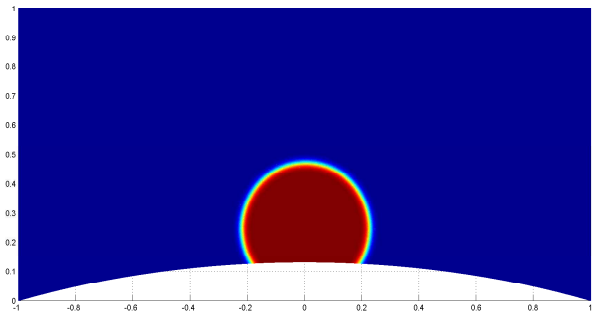

(d)numerical equilibrium states

Fig. 4.6.: The evolution of the thin film on the curved surface for prescribed contact angles $\theta_{s}=2 \pi / 3$.

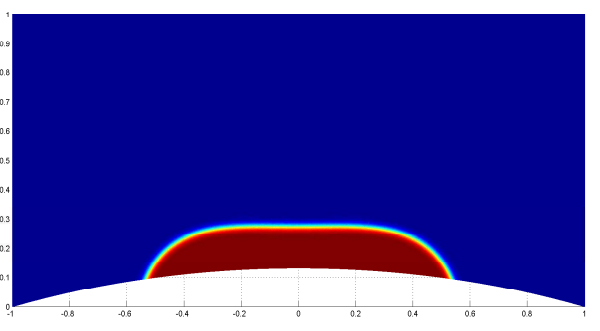

(a) $\mathrm{t}=25$

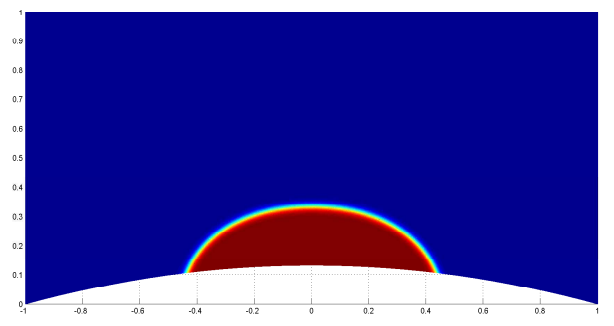

(c) $\mathrm{t}=200$

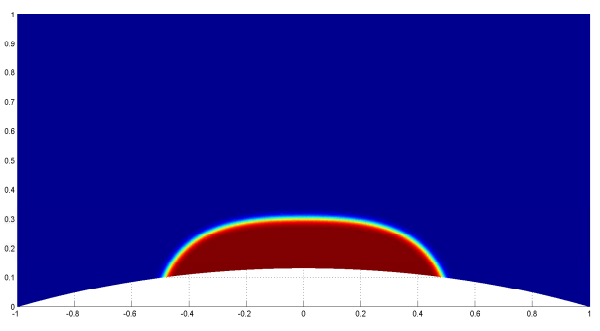

(b) $\mathrm{t}=100$

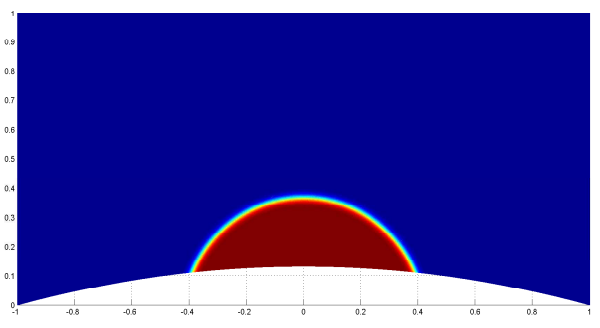

(d)numerical equilibrium states

Fig. 4.7.: The evolution of the thin film on the curved surface for prescribed contact angles $\theta_{s}=\pi / 3$.

[10] Chen, J., Sun, S., Wang, X.: A numerical method for a model of two-phase flow in a coupled free flow and porous media system. Journal of Computational Physics. 268, 1-16 (2014).

[11] J. Chen, S. Sun and Z. Chen. Coupling Two-Phase Fluid Flow with Two-Phase Darcy Flow in Anisotropic Porous Media. Advances in Mechanical Engineering, 2014, 6: 871021. 


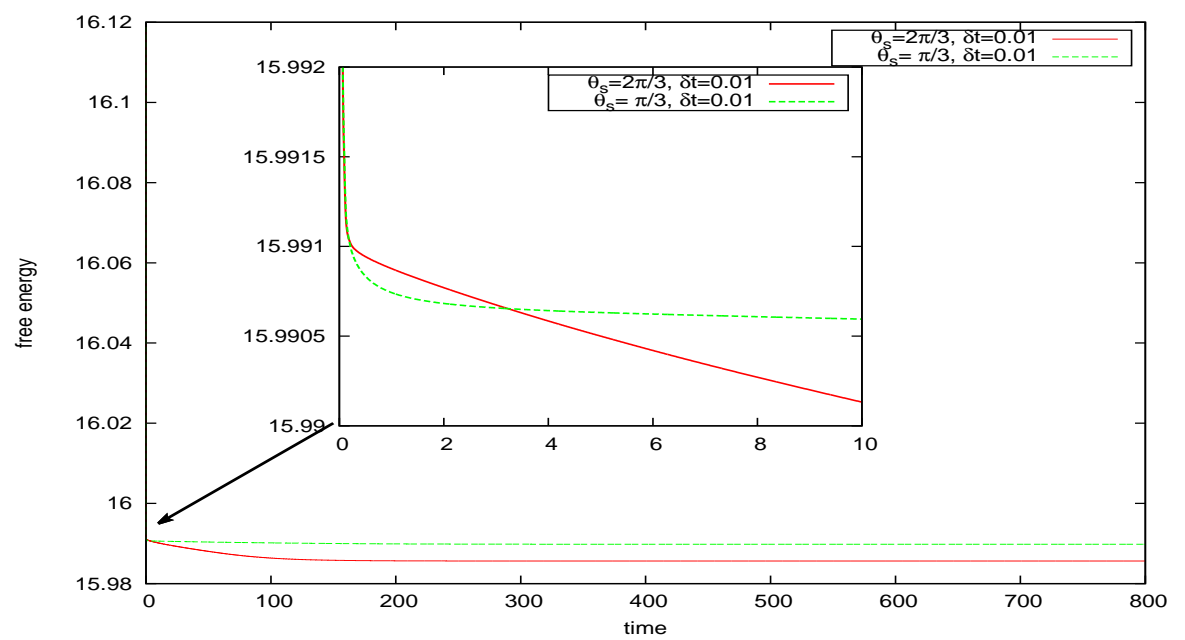

Fig. 4.8.: Time evolution of the free energy functional for two different prescribed contact angles $\theta_{s}=2 \pi / 3$ and $\theta_{s}=\pi / 3$ with time step $\delta t=0.01$. The energy curves show the decays for all prescribed contact angle, which confirms that our algorithm is unconditionally stable.

[12] Feng Bai, Xiaoming He, Xiaofeng Yang, Ran Zhou, and Cheng Wang. Three dimensional phasefield investigation of droplet formation in microfluidic flow focusing devices with experimental validation, International Journal of Multiphase Flow, 93: 130-141, 2017.

[13] Lee, H, G., Kim, J.: Accurate contact angle boundary conditions for the Cahn-Hilliard equations. Computers \& Fluids. 44, 178-186 (2011).

[14] Han, D., Brylev, A.: Numerical Analysis of Second Order, Fully Discrete Energy Stable Schemes for Phase Field Models of Two-Phase Incompressible Flows. J. Sci. Comput. 70, 965-989 (2017).

[15] Shen, J., Xu, J.: The scalar auxiliary variable (SAV) approach for gradient flows. Journal of Computational Physics. 353, 407-416 (2017).

[16] Shen, J., Xu, J.: a new class of efficient and robust energy stable schemes for gradient flows. SIAM Reviews (to appear)

[17] Shen J, Xu J. Convergence and error analysis for the scalar auxiliary variable (SAV) schemes to gradient flows[J]. SIAM Journal on Numerical Analysis, 2018, 56(5): 2895-2912.

[18] Jiran, E., Thompson, C, V.: Capillary instabilities in thin films. Journal of Electronic Materials. 19, 1153-1160 (1990).

[19] Jiran, E., Thompson, C, V.: Capillary instabilities in thin, continuous films. Thin Solid Films. 208, 23-28 (1992).

[20] Han, D., Wang, X.: A second order in time, uniquely solvable, unconditionally stable numerical scheme for Cahn-Hilliard-Navier-Stokes equation. Journal of Computational Physics. 290, 139-156 (2015).

[21] Guillengonzalez, F., Tierra, G.: On linear schemes for a Cahn-Hilliard diffuse interface model. Journal of Computational Physics. 234, 140-171 (2013).

[22] L, Rayleigh.: On the theory of surface forces. - II. Compressible fluids[J]. Philosophical Magazine Series 1. 33(201), 209-220 (1892).

[23] X. Yang, Linear, first and second order and Unconditionally Energy Stable Numerical Schemes for the Phase Field model of Homopolymer blends, 327:294-316, J. Comput. Phys., 2016. 
[24] J. Zhao, X. Yang, J. Li, and Q. Wang, Energy stable numerical schemes for a hydrodynamic model of Nematic liquid crystals, 38(5), A3264-A3290, SIAM. J. Sci. Comput., 2016.

[25] Yang, X., Ju, L.: Efficient linear schemes with unconditional energy stability for the phase field elastic bending energy model. Comput. Methods Appl. Mech. Engrg. 315, 691-712 (2017).

[26] Yang, X., Han, D.: Linearly first- and second-order, unconditionally energy stable schemes for the phase field crytal model. Journal of Computational Physics. 330, 1116-1134 (2017).

[27] Chen, L.: An integrated finite element methods package in matlab, technical report, University of California at Irvine, (2009)

[28] Jiang, W., Wang, Y.: Solid-State Dewetting and Island Morphologies in Strongly Anisotropic Materials. Scripta Materialia. 115, 123-127 (2016).

[29] Bao, W., Jiang, W.: A parametric finite element method for solid-state dewetting problems with anisotropic surface energies[J]. Journal of Computational Physics. 330, 380-400 (2017).

[30] Der Waals J D.: The thermodynamic theory of capillarity under the hypothesis of a continuous variation of density[J]. Journal of Statistical Physics. 20(2), 200-244 (1979).

[31] Kim, J., Kang, K.: Conservative multigrid methods for Cahn-Hilliard fluids. Journal of Computational Physics. 193, 511-543 (2004).

[32] Diegel, A, E., Wang, C.: Stability and Convergence of a Second Order Mixed Finite Element Method for the Cahn-Hilliard Equation[J]. Ima Journal of Numerical Analysis. 36(4), 1867-1897 (2016). 